\title{
Thyroid storm complicated by multisystem organ failure requiring plasmapheresis to bridge to thyroidectomy: A case report and literature review
}

\author{
Rabail Soomro, Natasha Campbell*, Stuart Campbell, Christopher Lesniak, Michael Sullivan, Raquel Ong, Jennifer Cheng and \\ Mohammad A Hossain \\ Department of Medicine, Jersey Shore University Medical Center, Hackensack Meridian Health, Neptune, USA
}

\begin{abstract}
Objective: Emphasizing awareness about the importance of prompt clinical identification, diagnosis, and updated medical management of thyroid storm.

Background: Thyroid storm is a life-threatening complication of hyperthyroidism, usually associated with Grave's disease but can be secondary to toxic multinodular goiter or solitary toxic adenomas. Thyroid storm is diagnosed by history, physical examination and laboratory data. Treatment options differ depending upon severity and include treatment of the precipitating event, thionamides, beta receptor blocking agents, bile acids sequestrants, steroids, high dose potassium iodide and in severe cases plasmapheresis. Here, we present a case of thyroid storm refractory to medical management requiring plasmapheresis to bridge to total thyroidectomy.

Case report: A 40-year-old man with a known history of Graves' disease and poor adherence to methimazole and propranolol who presented to the emergency department with tingling and numbness in bilateral upper extremities, shortness of breath and palpitations that had been progressively worsening over the past couple of months. Review of systems revealed weight loss of 150 pounds, bilateral swelling of his feet and ankles, bulging of his eyes and heat intolerance. Laboratory data at admission revealed TSH of $<0.01$. Initially, treated with conventional optimal medical therapy, but he still required plasmapheresis to ultimately bridge to total thyroidectomy.

Conclusions: Thyroid storm is one of the rare and lethal complications of untreated or undertreated Graves' disease with a mortality rate of up to 10-30\%. Potential catastrophic consequences of untreated Graves' disease include thyroid storm, shock and death. It is important for clinicians to be aware and knowledgeable of the diversity of presentation, severity of the conditions and updated management including plasmapheresis.
\end{abstract}

\section{Introduction}

Thyroid storm is a rare life-threatening medical emergency. It is associated with high mortality (10 to $30 \%)$ [1]. It is characterized by severe thyrotoxicosis and systemic hemodynamic decompensation. There are multiple risk factors but usually patients with Graves' disease are at a greater risk [1]. Patients can present with diverse signs and symptoms and varying degrees of organ decompensation. Treatment should be initiated promptly by targeting all steps of thyroid hormone synthesis, release and action. Patients who are not responding appropriately to medical therapy should be treated with therapeutic plasma exchange and later thyroidectomy once the patient is stabilized [2]. Here, we report a case of thyroid storm secondary to noncompliance with medication for Graves' disease which was managed appropriately in an intensive care unit (ICU) setting initially with medical therapy, then plasmapheresis and ultimately thyroidectomy.

\section{Case presentation}

A 40-year-old man with a known history of Graves' disease, who stopped taking his prescribed methimazole and propranolol for four months, presented to the emergency department with tingling and numbness in both of his upper extremities, shortness of breath and palpitations that had been progressively worsening over the past few months. Review of systems revealed that he had lost over 150 pounds in four years and has swelling of his feet and ankles, bulging of his eyes and heat intolerance. On presentation to the emergency department, initial vital signs were: blood pressure 118/64 $\mathrm{mmHg}$, heart rate $146 \mathrm{bpm}$, respiratory rate 17, oral temperature 98.1 degrees Fahrenheit, oxygen saturation $99 \%$ on room air. On physical examination, the patient was skinny, diaphoretic and in mild distress. Head and neck exam revealed proptosis, lid lag and a diffusely enlarged nontender thyroid without audible bruits. His pulse was irregular and he was tachycardic without an audible murmur. Lungs were clear to auscultation. There was nonpitting edema of the feet and ankles bilaterally. Tremors were visible upon outstretched arms. Neurologically, he was completely oriented and was able to provide a detailed history. Deep tendon reflexes were 3+ in both patellar and Achilles tendons. Laboratory values on admission and after treatment with methimazole for 24 hours are summarized below (Table 1). Chest radiograph showed cardiomegaly with clear lung fields. Echocardiogram showed systolic dysfunction with an ejection fraction of 30-35\%. He was started on propranolol and methimazole in the emergency department and was urgently seen by endocrinologist.

${ }^{\star}$ Correspondence to: Natasha Campbell, Internal Medicine Residency Program, Jersey Shore University Medical Center, Hackensack Meridian Health, Neptune, NJ 07753, Email: Natasha.campbell@hackensackmeridian.org

Key words: thyrotoxicosis, thyroid storm, plasmapheresis, grave's disease.

Received: October 31, 2019; Accepted: November 05, 2019; Published: November 08, 2019 
Table 1. Laboratory values on admission, after medical treatment and after plasmapheresis.

\begin{tabular}{|c|c|c|c|c|}
\hline LABS & On admission & $\begin{array}{c}\text { After } 24 \text { hours of medical } \\
\text { management }\end{array}$ & After plasmapheresis & Reference ranges \\
\hline Sodium (mmol/L) & 138 & 133 & 134 & $136-145$ \\
\hline Potassium (mmol/L) & 4.1 & 5.9 & 5.1 & $3.5-5.2$ \\
\hline $\mathrm{CO} 2(\mathrm{mmol} / \mathrm{L})$ & 26 & 16 & 20 & $24-31$ \\
\hline Anion gap & 3 & 14 & 8 & $8-16$ \\
\hline Glucose (mg/dL) & 103 & 79 & 169 & $70-110$ \\
\hline BUN (mg/dL) & 10 & 50 & 54 & $5-25$ \\
\hline Creatinine (mg/dL) & 0.87 & 2.22 & 1.71 & $0.61-1.24$ \\
\hline Phosphorous (mg/dL) & 4.8 & 7.2 & 5 & $2.5-4.6$ \\
\hline Calcium (mg/dL) & 9.1 & 8.3 & 8.5 & $8.5-10.5$ \\
\hline Albumin (g/dL) & 3.0 & 3.2 & 4 & $3.5-5$ \\
\hline Total bilirubin (mg/dL) & 3.5 & 9.6 & 7 & $0.2-1.3$ \\
\hline Alkaline Phosphatase (iU/L) & 382 & 407 & 194 & $38-126$ \\
\hline AST (iU/L) & 35 & 2439 & 2070 & $10-42$ \\
\hline ALT (iU/L) & 35 & 1473 & 1253 & $10-60$ \\
\hline LDH (iU/L) & - & 2368 & - & $91-200$ \\
\hline TSH (iU/mL) & $<0.01$ & - & - & $0.300-4.500$ \\
\hline Free T4 (NG/DL) & 5.23 & 4.54 & - & $0.50-1.26$ \\
\hline Free T3 $(\mathrm{pg} / \mathrm{mL})$ & - & 12.9 & - & $2.28-3.96$ \\
\hline WBC (K/uL) & 5.5 & 14.4 & 14.9 & $4.5-11.0$ \\
\hline Hemoglobin (gm/dL)/Hematocrit (\%) & $12.5 / 37.8$ & $14.1 / 41.8$ & $13.3 / 40.4$ & $\begin{array}{l}13.2-17.5 / \\
40.3-53.0\end{array}$ \\
\hline Platelet count $(\mathrm{K} / \mathrm{uL})$ & 146 & 89 & 40.4 & $140-450$ \\
\hline INR & - & 3.71 & 4.75 & $0.88-1.15$ \\
\hline Fibrinogen & - & - & 157 & $232-519$ \\
\hline Peripheral Smear & No schistocytes & No schistocytes & No schistocytes & - \\
\hline
\end{tabular}

The Burch-Wartofsky point scale for thyrotoxicosis was calculated to be 45 , which was highly suggestive of thyroid storm and he was started on methimazole, cholestyramine and intravenous hydrocortisone $100 \mathrm{mg}$ every eight hours. Within 24 hours, the patient developed altered mental status, lethargy, multisystem organ failure, disseminated intravascular coagulopathy and hyperkalemia. He was intubated for airway protection and transferred to intensive care unit. Potassium iodine was contraindicated due to hyperkalemia and methimazole was discontinued due to liver failure. Lithium was considered as a treatment option, however due to his rapid decline and worsening renal function, lithium was not started and the decision was made to urgently place a vascular catheter and proceed with plasmapheresis. Two sessions of plasmapheresis were performed. After plasmapheresis, the renal and liver function were improved. Meanwhile, the patient was evaluated by an endocrine surgeon for thyroidectomy. His platelet count and international normal ratio (INR) stabilized and the patient's neurological function improved on the ventilator after plasma exchange. He was successfully extubated and was taken to surgery on following day. After thyroidectomy, levothyroxine and calcium supplementation were started. Cholestyramine was discontinued, and hydrocortisone was tapered. Still, atrial fibrillation with rapid ventricular response continued requiring continuous diltiazem infusion. He underwent transesophageal echocardiogram (TEE) with cardioversion and was successfully converted to normal sinus rhythm. He was transitioned to an oral regimen of diltiazem and propranolol by cardiology. He was discharged with the instruction to continue apixaban for anticoagulation for one month as per cardiologist.

\section{Discussion}

Thyroid storm is the most severe form of thyrotoxicosis. It is a hypermetabolic condition associated with significant morbidity and mortality. At times, diagnosis can be challenging due to the diversity of symptoms specifically in younger patients or patients with altered mental status, which can be mistaken for substance abuse [3]. Thyroid hormone is the key regulating hormone in our body's metabolism. It increases tissue thermogenesis and our basal metabolic rate. Triiodothyronine (T3) and thyroxine (T4) have widespread multiorgan effects [3]. Younger patients tend to present with symptoms of sympathetic stimulation such as anxiety, restlessness and tremor, whereas older patients tend to present with less obvious clinical manifestations. Elderly patients may lack adrenergic symptoms and report depression, fatigue and weight loss, which is termed apathetic hyperthyroidism [3]. Cardiovascular symptoms include tachycardia that can exceed 140 beats/minute resulting in high output systolic heart failure. Hypotension, cardiac arrhythmias and death from cardiovascular collapse may also occur [4]. A variety of atrial and ventricular tachycardias have been described in hyperthyroidism, but the most common arrhythmia is atrial fibrillation [5]. Hyperpyrexia ranging from $104-106^{\circ} \mathrm{F}$ is common due to the overall hypermetabolic state. Agitation, anxiety, delirium, psychosis, stupor or coma are also seen and are considered by many to be essential to the diagnosis. Other symptoms may include hepatic failure with jaundice [6-8].

Graves' disease, toxic multinodular goiter or solitary toxic adenomas are associated with a hyperthyroid state [9]. If left untreated, thyroid storm can develop in these patients. Among these, thyroid storm associated with Graves' disease is most reported [10] and is generally precipitated by an event such as surgery, trauma, infection or parturition [11]. Poor compliance with antithyroid medications is a commonly reported precipitant of thyroid storm associated with Graves' disease.

The diagnosis of thyroid storm is based upon the presence of symptoms with biochemical evidence of hyperthyroidism i.e. elevation of free T4 and/or T3 and suppression of thyroid stimulating hormone (TSH). In 1993, Burch and Wartofsky introduced a scoring system using precise clinical criteria for the identification of thyroid storm [12]. 
A score of 45 or more is highly suggestive of thyroid storm whereas a score below 25 makes thyroid storm unlikely. A score of 25 to 44 is suggestive of impending storm. In our patient, the Burch Wartofsky score was $45[9,12]$.

Literature review revealed few cases of thyroid storm requiring plasmapheresis. Carhill et al. reported two severe cases of thyroid storm with Burch-Wartofsky scores of 80 and 70 respectively. Presenting complaints varied slightly. One patient reported palpitations, nausea, vomiting and dyspnea, whereas the other patient also had worsening lower extremity edema. Our patient presented with symptoms of numbness and tingling in his upper extremities, which is not typically seen. Another differentiating point in our patient was the development of altered mental status. These patients also developed high output systolic heart failure and cardiac arrhythmias. Interestingly, treatment with methimazole caused worsening transaminitis in both patients. While one of their patients was briefly able to tolerate treatment with lithium, medical treatment ultimately failed and plasmapheresis with eventual total thyroidectomy was indicated like our patient $[6,13]$.

Plasmapheresis rapidly decreases the concentration of harmful constituents such as antibodies, immune complexes and toxins and has been used historically for myasthenia gravis and rheumatoid arthritis $[6,7,14]$. The patient's plasma is replaced with a colloid solution of choice, which varies in different institutions, but usually includes fresh frozen plasma (FFP) and albumin. Thyroid binding globulin (TBG), which is bound with thyroid hormone is removed in this process and the albumin serves as a new binding site for freely circulating thyroid hormone $[6-8,13,14]$.

In 1970, Ashkar et al. first described the use of therapeutic plasma exchange (TPE) in treating thyrotoxic crisis. After serum thyroxine levels were reduced by $40 \%$ in a dog with hyperthyroid-like state, three separate patients were treated with plasmapheresis after failed medical management when mortality appeared imminent. Rapid and effective improvement was seen. In 2009, Ezer et al. reported a case series including 11 patients with thyrotoxicosis who were treated with plasmapheresis prior to surgery [7,14]. All 11 patients showed improvement in their presenting symptoms and rapid normalization of circulating thyroid hormone levels.

In planned or emergent thyroidectomy as the treatment option for thyroid storm secondary to Graves' disease, proper preparation is ideal. Patients should be rendered euthyroid with methimazole if tolerated. Beta blockers, corticosteroids and saturated solution of potassium iodide (SSKI) should be given immediately preoperatively. These treatment modalities have been found to decrease thyroid blood flow, vascularity and overall intraoperative blood loss. An interdisciplinary care plan should be made between the critical care team, endocrinology, thyroid surgeon and anesthesiologist to try to obtain euthyroid state prior to surgery. High volume thyroid surgery (over 30 thyroid surgeries performed annually) has been found to have statistically significant better outcomes [15]. Complications such as permanent hypocalcemia, permanent recurrent laryngeal nerve damage and bleeding requiring reoperation are seen $<2 \%$ of the time [15-17].

If thyroid storm is suspected, high acuity level care in the ICU is required to ensure prompt thyrotoxicosis-directed therapy, treatment of any precipitant illness and supportive measures. Thyrotoxicosis is treated with a multidrug approach including intravenous $\beta$-blockers, thionamides (propylthiouracil (PTU) and methimazole), intravenous high-dose glucocorticoids, and potassium iodide. Glucocorticoid therapy is a potent inhibitor of peripheral T4 to T3 conversion and is hypothesized to treat relative adrenal insufficiency. Bile acid sequestrants such as cholestyramine can also be used to decrease T4 and T3 levels $[8,14]$. Potassium iodide (KI) inhibits thyroid hormone secretion, however potassium levels must be closely monitored with serial serum metabolic panels. In some cases, lithium can be used but due to its low therapeutic index and potential renal and neurologic adverse effects it is not the preferred choice. Our patient had elevated liver function tests (LFTs) and renal failure, which rendered thionamides and lithium unusable. As in our case, plasmapheresis is a successful option in patients who respond poorly to conventional medical therapy in order to bridge toward definitive therapy. Definitive treatment with thyroidectomy or 131I therapy is indicated in patients who survive thyroid storm $[8,9,11,17,18]$.

\section{Conclusion}

The diagnosis of thyroid storm can be overlooked due to the varying presentations, which can lead to serious consequences. Therefore, diagnosing a patient in a timely manner with adequate management is crucial to minimize the associated mortality. Treatment should be started immediately in ICU setting. A multidrug approach is the most effective way of treatment. Therapeutic plasma exchange should be considered earlier in patients who are not responding to optimum medical therapy and the internist should be aware of this option. In patients with thyroid storm secondary to uncontrolled Graves' disease, definitive treatment options such as iodine ablation and total thyroidectomy should be considered to prevent recurrence.

\section{Author Contributions}

R.S (Rabail Soomro), N.C (Natasha Campbell), S.C(Stuart Campbell), C.L(Christopher Lesniak) were involved in the case selection, in addition to planning and drafting the manuscript.

R.S (Rabail Soomro), N.C (Natasha Campbell), M.S(Michael Sullivan)- Were involved in the case discussion.

R.O(Raquel Ong), J.C(Jennifer Cheng)- worked on the case discussion, case presentation and discussion.

M.A.H (Mohammad A. Hossain) - worked on the planning, manuscript revision and final approval.

\section{References}

1. Sanna Fatima, Ritika Puri, Soumya Patnaik, Jorge Mora (2016) When toxic thyroid makes liver toxic a case of thyroid storm complicated by acute liver failure. AACE clinical case reports $3: 3$.

2. Chiha M, Samarasinghe S, Kabaker AS (2015) Thyroid Storm: An Updated Review. $J$ Intensive Care Med 30: 131-140. [Crossref]

3. Danielle Devereaux, Semhar Z, Tewelde (2014) Hyperthyroidism and Thyrotoxicosis. Emerg Med Clin North Am 32: 277-292. [Crossref]

4. Su Yin Adeline Ngo, Huck Chin Chew (2007) When the storm passes unnoticed-A case series of thyroid storm. Resuscitation 73: 485-490.

5. Toft AD, Boon NA (2000) Thyroid disease and the heart. Heart 84: 455-460.

6. Carhill A, Gutierrez A, Lakhia R (2012) Surviving the storm: two cases of thyroid storm successfully treated with plasmapheresis. BMJ Case Reports 2012: bcr2012006696. [Crossref]

7. Ashkar FS, Katims RB, Smoak WM, Gilson AJ (1970) Thyroid storm treatment with blood exchange and plasmapheresis. JAMA 214: 1275-1279. [Crossref]

8. Rivkees SA, Szarfman A (2010) Dissimilar hepatotoxicity profiles of propylthiouracil and methimazole in children. J Clin Endocrinol Metab 95: 3260-3267. [Crossref]

9. Waltman PA, Brewer JM, Lobert S(2004) Thyroid Storm During Pregnancy A Medical Emergency. Crit Care Nurse 24: 74-79. [Crossref] 
10. Simsir IY, Ozdemir M, Duman S, Erdogan M, Donmez A, et al. (2018) Therapeutic plasmapheresis in thyrotoxic patients. Endocrine 62: 144-148. [Crossref]

11. Erbil Y, Ozluk Y, Giriș M, Salmaslioglu A, Issever H, et al. (2007) Effect of Lugol solution on thyroid gland, blood flow and microvessel density in patients with Graves' disease. J Clin Endocrinol Metab 92: 2182-2189. [Crossref]

12. Burch HB, Wartofsky L (1993) Life-threatening thyrotoxicosis, thyroid storm. Endocrinol Metab Clin North Am 22: 263-277. [Crossref]

13. Baena JC, Padilla J, Guzmán G (2017) Thyroid storm associated with multiorganic dysfunction. Medicina (B Aires) 77: 337-340. [Crossref]

14. Ezer A, Caliskan K, Parlakgumus A, Belli S, Kozanoglu I, et al. (2009) Preoperative therapeutic plasma exchange in patients with thyrotoxicosis. J Clin Apher 24: 111-114. [Crossref]
15. Röher HD, Goretzki PE, Hellmann P, Witte J (1999) Complications in thyroid surgery; Incidence and therapy. Chirurg 70: 999-1010. [Crossref]

16. Cooper DS (2005) Antithyroid drugs. N Engl J Med 352: 905.

17. Watanabe N, Narimatsu H, Noh JY (2012) Antithyroid drug-induced hematopoietic damage: a retrospective cohort study of agranulocytosis and pancytopenia involving 50,385 patients with Graves' disease. J Clin Endocrinol Metab 97: E4953. [Crossref]

18. Bahn RS, Burch HS, Cooper DS (2009) The Role of propylthiouracil in the management of Graves' disease in adults: report of a meeting jointly sponsored by the American Thyroid Association and the Food and Drug Administration. Thyroid 19: 673-674. [Crossref]

Copyright: (C2019 Soomro R. This is an open-access article distributed under the terms of the Creative Commons Attribution License, which permits unrestricted use, distribution, and reproduction in any medium, provided the original author and source are credited. 